\title{
Treatment of inclusion body myositis: is low-dose intravenous immunoglobulin the solution?
}

\author{
Mike Recher · Ulrike Sahrbacher · Juliane Bremer • \\ Börge Arndt · Urs Steiner · Adriano Fontana
}

Received: 25 September 2009 / Accepted: 29 November 2009 / Published online: 1 January 2010

(C) Springer-Verlag 2009

\begin{abstract}
Inclusion body myositis (IBM), the most common inflammatory myopathy in the elderly, is often resistant to various forms of therapy. Placebo-controlled treatment trials with high dose intravenous immunoglobulins (IVIG) have shown disease amelioration in some but not all patients. Here, we present the informative case of a 70-year-old woman with diagnosed inclusion body myositis that showed progressive muscle weakness without treatment and following immuno-suppressive treatment with corticosteroids and azathioprine. A trial with low-dose intravenous immunoglobulins was started at that time. The patient responded rapidly to low dose IVIG treatment with amelioration of muscle strength and normalization of CK serum activities. Our results demonstrate that IBM patients may respond to low-dose IVIG treatment which has important clinical and economic consequences.
\end{abstract}

Keywords IBM $\cdot$ IVIG $\cdot$ Myositis $\cdot$ Inclusion body myositis · Low-dose IVIG

$\begin{array}{ll}\text { Abbreviations } \\ \text { IVIG } & \text { Intravenous immunoglobulins } \\ \text { IBM } & \text { Inclusion body myositis } \\ \text { CK } & \text { Creatin-kinase }\end{array}$

M. Recher and U. Sahrbacher contributed equally to this work.

M. Recher $(\varangle) \cdot$ U. Sahrbacher · B. Arndt · U. Steiner · A. Fontana Clinic for Immunology, Department of Internal Medicine,

University Hospital Zürich, Haeldeliweg 4,

8044 Zurich, Switzerland

e-mail: mike.recher@usz.ch; mike.recher@childrens.harvard.edu

J. Bremer

Institute of Neuropathology, Department of Pathology,

University Hospital Zurich, Schmelzbergstrasse 12,

8091 Zurich, Switzerland

\section{Introduction}

Inclusion body myositis (IBM) is the most frequent inflammatory myopathy in the elderly [1]. The disease typically progresses slowly and involves mostly the quadriceps muscles, arm flexors and small muscles of the fingers. Spontaneous stabilization of disease activity has been reported in the absence of any therapy [2]. Characteristic microscopic findings are typical intramuscular vacuoles and foci of amyloid as well as immunological features including MHC I up-regulation and oligoclonally proliferated infiltrating $\mathrm{CD}^{+} \mathrm{T}$ cells [3]. In general, response to any treatment of IBM is weak [2]. Placebo-controlled studies showed limited effects of high dose ( $2 \mathrm{~g} / \mathrm{kg})$ IVIG therapy in IBM [4, 5]. It has been noted that at least in some patients progression of disease stopped while others did not respond, resulting in a mild overall effect of IVIG therapy [5]. As a consequence, Pongratz et al. [6] suggested a 3-month trial of high-dose $(2 \mathrm{~g} / \mathrm{kg} /$ month $)$ IVIG to identify IVIG responders among IBM patients.

\section{Case report}

A 70-year-old woman was evaluated for progressive muscle weakness and mildly elevated serum creatin-kinase (CK) activity. CK elevation and muscle weakness continued following cessation of treatment with a HMG-Co A reductase inhibitor. Muscle weakness mostly affected strength in the quadriceps muscles. There were enhanced signal intensities in water-sensitive MRI sequences of the adductor magnus muscle in both legs and of the semimembranous muscle in the right leg (not shown). Biopsy of the right $M$. adductus magnus showed vacuoles in several muscle fibers; some of them appeared as typical 'rimmed 
Fig. 1 a Histology of the patient's muscle biopsy (M. adductor magnus). Trichrome staining shows a rimmed vacuole. Immunohistochemistry demonstrates upregulation of MHC class I expression on several muscle fibers, and endomysial infiltrates of CD45

(leukocyte common antigen) positive leukocytes. b Serum creatin-kinase (CK) activities are shown. $\mathbf{c}$ Muscle strength of knee extension on the right leg is indicated at individual timepoints under different therapies a

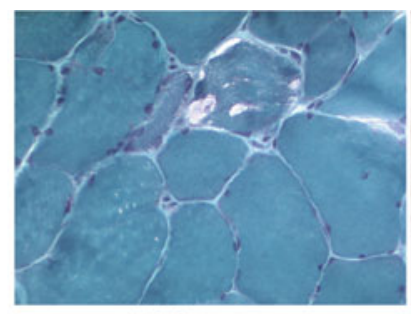

Trichrom

b

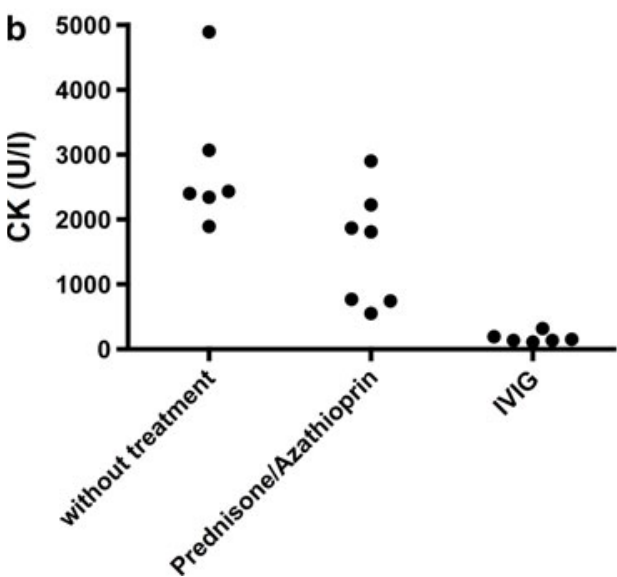

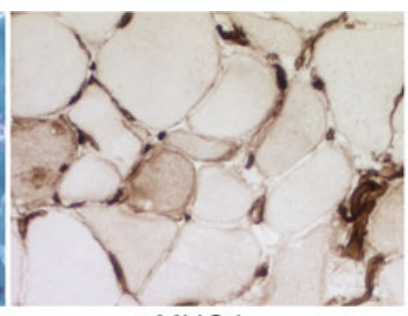

$\mathrm{MHC}$ I

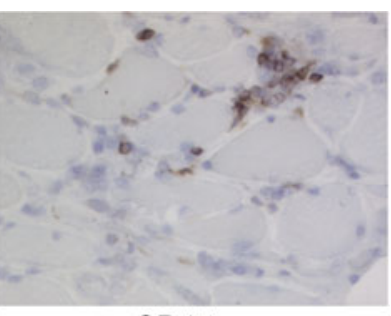

CD45

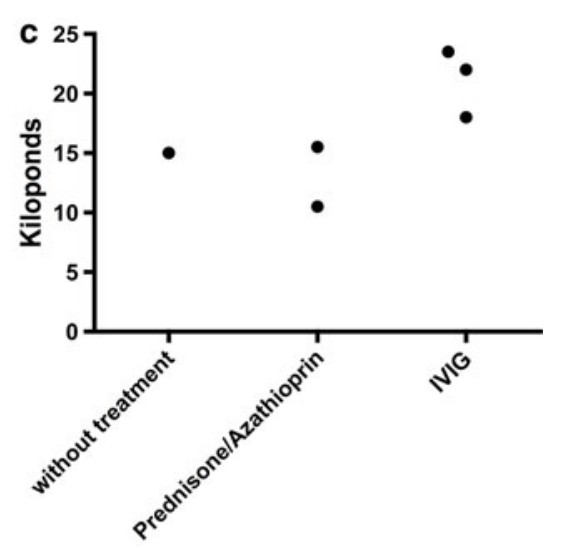

vacuoles'. MHC class I expression was up-regulated on several muscle fibers (Fig. 1a). Together with infiltrating leukocytes (Fig. 1a), histologic findings were most consistent with inclusion body myositis (IBM). Histological alterations indicative of statin-induced myopathy were not found. Myositis-related serum autoantibodies (Anti Jo-1, Anti-PM SCL, Anti PL-7, Anti PL-12, Anti-Ku, Anti Mi-2) were not detected. There was no clinical evidence of viral- or superimposed systemic autoimmune disease. The diagnosis of IBM was established. Due to the reported very limited efficacy of any immune-modulating therapy and the reported spontaneous stabilization of IBM disease, no medication was started at that time, and the disease followed clinically.

However, muscle weakness progressed. The patient was finally unable to leave the house alone and became increasingly depressed. In the end, hospitalization was required due to intake of a neuroleptic drug with the purpose of suicide.

A therapeutic trial with prednisone/azathioprine (given at usual doses, prednisone initially $1 \mathrm{mg} / \mathrm{kg}$ with tapering thereafter, azathioprine $2 \mathrm{mg} / \mathrm{kg}$ ) was started at that time, but it showed no effect on quality of life and muscle strength, although it was associated with mildly decreased CK serum activity (Fig. 1b). Prednisone/azathioprine treatment was stopped after 6 months due to lack of subjective and objective signs of improvement. A trial of intravenous immunoglobulins (IVIG) was initiated at that time.
In the light of rather disappointing effects of IVIG in recent studies in IBM (see below), we reduced the standard trial dose of IVIG from 2 to $0.3 \mathrm{~g} / \mathrm{kg} /$ day given on two consecutive days as a monthly cycle $(0.6 \mathrm{~g} / \mathrm{kg}$ IVIG per month). The reason for using a lower than normal IVIG dose was mostly to reduce IVIG-related side effects in light of the low probability of clinical success. Within days after the first infusion, there was an unexpected rapid subjective amelioration of the muscle strength and trend to amelioration at the objective level over the next months (Fig. 1c). Elevated muscle enzymes normalized within 1 month (Fig. 1b) and since then without exception stay within normal range for now 12 months after initiation of IVIG therapy. Control MRI scans of the leg muscles now failed to detect active myositis (not shown). Quality of life improved remarkably, and the patient remains in remission for now 12 months after initiation of IVIG therapy.

\section{Discussion}

The rapid response following initiation of low-dose IVIG treatment in this patient was highly unlikely representing spontaneous disease course since the patient was before followed clinically for several months without any medication; however, muscle weakness and general condition progressed. Also, the negative response to a prednisone/ azathioprine trial at usual dosage (initially $1 \mathrm{mg} / \mathrm{kg}$ Prednisone 
and $2 \mathrm{mg} / \mathrm{kg}$ azathioprine) further excludes other types of inflammatory muscle disease that would clinically respond to this standard immuno-suppressive regimen. High IVIG doses require long infusion time or a higher infusion rate, which is associated with IVIG-related side effects, such as fever, chills, headache or skin reactions [7]. For patients weighing $80 \mathrm{~kg}, 2 \mathrm{~g} / \mathrm{kg}$ IVIG equals $160 \mathrm{~g}$ IVIG which would require infusion of 13 large ampoules ( $12 \mathrm{~g}$ each) of a common IVIG product. This reflects an infusion volume of 2.61 and costs of approximately 10,000 US dollars per month. Thus, reducing the IVIG dose reduces volume load, infusion time, IVIG-related side effects and medication costs.

The immune-suppressive mechanism of high-dose IVIG is best documented in idiopathic thrombocytopenic purpura (ITP), where a transient inability of macrophages to opsonize immune complexes following IVIG treatment has been demonstrated $[8,9]$. In recent publications, especially the group of Ravetch [10] has shed new light to the immunesuppressive effects of high dose IVIG. First, IVIG acts by means of the inhibitory IgG receptor. Second, the immunesuppressive activity is restricted to a small proportion of $\mathrm{IgG}$ whose $\mathrm{Fc}$ fragment is sialylated [11]. Sialylated IgG-Fc can be recombinantly produced and is much more effective on a molar basis than conventional IVIG [12]. Finally, sialylated IVIG mediates its effects by binding to a receptor called DC-Sign in humans [13]. IVIG treatment has been associated with normalization of complement activation in patients with dermatomyositis [14]. How IVIG may improve myositis in IBM patients that respond to the treatment is completely unknown. A study analyzing specifically immune-modulating effects of IVIG on muscle infiltrating cells and their respective cytokines demonstrated very limited effects in inflammatory muscle diseases [15]. Myocytes have been shown to express B7-H3, which might protect myocytes from $\mathrm{CD} 8^{+} \mathrm{T}$ cell-mediated lysis [16]. In contrast to many autoimmune conditions, TNF blockade was associated with high incidence of disease flares in inflammatory myopathies [17].

In our patient, IVIG treatment was unexpectedly successful in a low-dose regimen which costs approximately fourfold less than high-dose IVIG. The very rapid decrease of CK serum activity following the first IVIG application after a reasonable period of no therapy and a trial with prednisone/azathioprine make it unlikely that the amelioration was reflecting spontaneous disease course. In addition, a similar long lasting and unexpectedly good response to a comparable low-dose IVIG trial has been reported in an IBM patient [18]. It may be asked whether high dose IVIG is counterproductive because of temporarily increased blood viscosity and/or direct toxic effects of immunoglobulins on the already abnormal myocytes in IBM. Alternatively, the few patients that positively respond to IVIG therapy in the high dose IVIG trials would probably also respond to much lower IVIG doses. This would have important implications for economic reasons and would likely help reducing IVIG related side effects. Taken collectively, we propose to conduct a novel prospective controlled clinical trial of the effectiveness of low-dose IVIG therapy in IBM patients.

Conflict of interest statement The authors report no conflicts of interest.

\section{References}

1. Dalakas MC (1991) Polymyositis, dermatomyositis and inclusionbody myositis. N Engl J Med 325(21):1487-1498

2. Griggs RC (2006) The current status of treatment for inclusionbody myositis. Neurology 66(2 Suppl 1):S30-S32

3. Needham M, Mastaglia FL (2007) Inclusion body myositis: current pathogenetic concepts and diagnostic and therapeutic approaches. Lancet Neurol 6(7):620-631

4. Dalakas MC, Sonies B, Dambrosia J, Sekul E, Cupler E, Sivakumar K (1997) Treatment of inclusion-body myositis with IVIg: a double-blind, placebo-controlled study. Neurology 48(3):712-716

5. Walter MC, Lochmuller H, Toepfer M, Schlotter B, Reilich P, Schroder M et al (2000) High-dose immunoglobulin therapy in sporadic inclusion body myositis: a double-blind, placebo-controlled study. J Neurol 247(1):22-28

6. Pongratz D (2006) Therapeutic options in autoimmune inflammatory myopathies (dermatomyositis, polymyositis, inclusion body myositis). J Neurol 253(Suppl 5):V64-V65

7. Wittstock M, Benecke R, Zettl UK (2003) Therapy with intravenous immunoglobulins: complications and side-effects. Eur Neurol 50(3):172-175

8. Imbach P, Barandun S, d'Apuzzo V, Baumgartner C, Hirt A, Morell A et al (1981) High-dose intravenous gammaglobulin for idiopathic thrombocytopenic purpura in childhood. Lancet 1(8232):1228-1231

9. Bussel JB, Kimberly RP, Inman RD, Schulman I, CunninghamRundles C, Cheung N et al (1983) Intravenous gammaglobulin treatment of chronic idiopathic thrombocytopenic purpura. Blood 62(2):480-486

10. Samuelsson A, Towers TL, Ravetch JV (2001) Anti-inflammatory activity of IVIG mediated through the inhibitory Fc receptor. Science 291(5503):484-486

11. Kaneko Y, Nimmerjahn F, Ravetch JV (2006) Anti-inflammatory activity of immunoglobulin $\mathrm{G}$ resulting from $\mathrm{Fc}$ sialylation. Science 313(5787):670-673

12. Anthony RM, Nimmerjahn F, Ashline DJ, Reinhold VN, Paulson JC, Ravetch JV (2008) Recapitulation of IVIG anti-inflammatory activity with a recombinant IgG Fc. Science 320(5874):373-376

13. Anthony RM, Wermeling F, Karlsson MC, Ravetch JV (2008) Identification of a receptor required for the anti-inflammatory activity of IVIG. Proc Natl Acad Sci USA 105(50):19571-19578

14. Dalakas MC (2006) The role of high-dose immune globulin intravenous in the treatment of dermatomyositis. Int Immunopharmacol 6(4):550-556

15. Barbasso Helmers S, Dastmalchi M, Alexanderson H, Nennesmo I, Esbjornsson M, Lindvall B et al (2007) Limited effects of highdose intravenous immunoglobulin (IVIG) treatment on molecular expression in muscle tissue of patients with inflammatory myopathies. Ann Rheum Dis 66(10):1276-1283 
16. Waschbisch A, Wintterle $\mathrm{S}$, Lochmuller $\mathrm{H}$, Walter $\mathrm{MC}$, Wischhusen J, Kieseier BC et al (2008) Human muscle cells express the costimulatory molecule $\mathrm{B} 7-\mathrm{H} 3$, which modulates muscle-immune interactions. Arthritis Rheum 58(11):3600-3608

17. Dastmalchi M, Grundtman C, Alexanderson $\mathrm{H}$, Mavragani CP, Einarsdottir H, Helmers SB et al (2008) A high incidence of disease flares in an open pilot study of infliximab in patients with refractory inflammatory myopathies. Ann Rheum Dis 67(12):1670-1677

18. Mukunda BN, Dileep Kumar P, Smith HR (2001) Long-lasting effectiveness of intravenous immunoglobulin in a patient with inclusion-body myositis. Ann Intern Med 134(12):1156 